\title{
Antithrombotic Cancer Associated Thrombosis: It May Change
}

Andrea Fontanella ${ }^{1}$, Mauro Campanini ${ }^{2}$, Francesco Dentali ${ }^{3}$, Gualberto Gussoni ${ }^{4 *}$, Dario Manfellotto ${ }^{5}$, Mauro Silingardi ${ }^{6}$, Giancarlo Agnelli ${ }^{7}$ and Pierpaolo Di Micco'

${ }^{1}$ Department of Internal Medicine, Fatebenefratelli Hospital, Napoli, Italy

2Department of Internal Medicine, Hospital 'Maggiore della Carità', Novara, Italy

${ }^{3}$ Department of Internal Medicine, Hospital 'Luini Confalonieri', Luino, Italy

${ }^{4}$ Research Department, FADOI Foundation, Milano, Italy

${ }^{5}$ Department of Internal Medicine, Fatebenefratelli Hospital-AFaR, Roma, Italy

${ }^{6}$ Department of Internal Medicine A, Maggiore Hospital, Bologna, Italy

${ }^{7}$ Department of Internal and Cardiovascular Medicine-Stroke Unit, University of Perugia, Perugia, Italy

\begin{abstract}
Active treatment of acute venous thromboembolism without active bleeding is primarily based on the administration of $\mathrm{LMWH}$, according to international guidelines. However, recent studies have provided data on direct oral anticoagulants that offer interesting new perspectives concerning efficacy in the prevention of recurrent VTE and the safety of these drugs in this particular patient population.
\end{abstract}

Keywords: VTE; Venous thromboembolism; Rivaroxaban

\section{Background}

For several years, the suggested and recommended antithrombotic therapy for VTE in cancer was based on prolonged administration of low-molecular-weight heparins (LMWH), according to ASCO, NCCN and other international guidelines [1]. Several reports based on real-life data have confirmed that this kind of treatment is safe in terms of the rates of VTE recurrence and/or bleeding [2].

Oral treatment for VTE in cancer is less common and has been tested in a few studies with warfarin; however, there are some issues with the use of anti-vitamin $\mathrm{K}$ drugs like warfarin in this clinical setting, due to the many drug-drug and drug-food interactions and to the relative difficulty of obtaining a stable INR with both fixed and adjusted doses of warfarin [3].

Even so, oral treatment for VTE in cancer is currently being debated now that direct oral anticoagulants (DOACs) have become available. These drugs are, in fact, on the market with an indication for treatment of venous thromboembolism, but the percentage of patients enrolled in the pivotal trials who had cancer was low (around $5 \%$ ). However, DOACs have a more predictable pharmacokinetic profile and less interaction with other drugs or food compared to vitamin $\mathrm{K}$ antagonists (VKAs), and therefore they are of potential interest for patients with cancer [4]. Consequently, new direct oral anticoagulants were tested in recent clinical trials that focused on improving our understanding of their possible use in this setting. Here we report the data available on the use of traditional treatments and DOACs in cancer patients with VTE, taken from the most frequently-referenced studies in the literature.

\section{Traditional Treatments}

\section{Warfarin}

Classically, treatment for acute VTE has been based on initial therapy with unfractionated heparin or LMWH, followed by long-term treatment with VKAs such as warfarin. The same treatment regimen was used for decades in oncology patients with VTE, despite the absence of specific studies in this setting [3]. Unfortunately, in previous years most cancer patients showed reduced tolerance to warfarin, particularly during chemotherapy, because of its frequent association with gastrointestinal and haematologic side effects. For this reason, it was very difficult to maintain therapeutic levels of warfarin, and one third of treated patients experienced VTE recurrence or treatmentrelated bleeding $[5,6]$.

\section{Low molecular weight heparins (LMWHs)}

All scientific communities have emphasised, in their international guidelines, a particular recommendation for low-molecular-weight heparin as treatment for patients with cancer-associated thrombosis (CAT), particularly VTE. The most important evidence for this recommendation came from randomised clinical trials such as the CLOT study and the CATCH study.

\section{CLOT study}

The CLOT study may be considered a milestone, because it was the first study designed to compare LMWH vs warfarin for the treatment of VTE during cancer. Until the CLOT study, patients with cancer and VTE were given similar treatment to non-oncological patients with VTE [7]; the initial treatment with LMWH was considered only as a bridge before continuing with warfarin for the long-term treatment of VTE. Most common issues raised in clinical practice and in scientific debates were based on the more predictable pharmacokinetics and reduced drug-drug interaction of $\mathrm{LMWH}$, which could provide clinical benefits compared to VKA in oncology patients; these discussions were also based on the fact that these patients often undergo complex treatment regimens and frequently experience liver dysfunction and malnutrition.

In the CLOT study, patients with VTE and malignancy were assigned, after randomisation, to receive long-term treatment with

*Corresponding author: Gualberto Gussoni, Research Department, FADO Foundation, Piazza Cadorna, Milano, Italy, Tel: +390285355188; E-mail: gualberto.gussoni@gmail.com

Received December 20, 2018; Accepted December 25, 2018; Published December 29, 2018

Citation: Fontanella A, Campanini M, Dentali F, Gussoni G, Manfellotto D, et al. (2018) Antithrombotic Cancer Associated Thrombosis: It May Change. J Blood Lymph S1: 007. doi:10.4172/2165-7831.S1-007

Copyright: (c 2018 Fontanella A, et al. This is an open-access article distributed under the terms of the Creative Commons Attribution License, which permits unrestricted use, distribution, and reproduction in any medium, provided the original author and source are credited. 
warfarin or with dalteparin $200 \mathrm{IU} / \mathrm{kg}$ daily for the first month, followed by a daily dose of approximately $150 \mathrm{IU} / \mathrm{kg}$ for the next five months. In this study, dalteparin showed its superiority over warfarin for preventing recurrent VTE in oncology patients. Concerning safety, no significant differences in major bleeding were found between dalteparin and warfarin.

\section{CATCH study}

The CATCH study was an open-label, randomised clinical trial that compared tinzaparin vs. warfarin as long-term anticoagulant therapy for VTE in oncology patients [8]. For this study, 900 patients with CAT were selected and randomised to receive tinzaparin $175 \mathrm{IU} / \mathrm{kg}$ once daily for 6 months or tinzaparin $175 \mathrm{IU} / \mathrm{kg}$ once daily for 5-10 days followed by warfarin at a dose adjusted to maintain an international normalized ratio target of 2.0-3.0 for 6 months. The primary objective was to evaluate the efficacy of tinzaparin for VTE recurrence in patients with active cancer; the secondary objective was to determine the safety of long-term treatment with tinzaparin (i.e., 6 months) in this clinical setting.

The results showed that the patients randomised to tinzaparin had a lower rate of recurrence of VTE compared to the warfarin group, although this was not statistically significant. On the other hand, concerning safety, there were no differences in the frequency of major bleeding or overall mortality between the two groups, while a significant reduction in clinically relevant non-major bleeding (CRNMB) was observed in those patients treated with tinzaparin [8].

\section{Fondaparinux}

There are no specific clinical trials that have tested the efficacy and safety of fondaparinux for the treatment of VTE in oncology; however, clinical data on this topic are available from a subgroup analysis of the MATISSE international clinical trial on VTE [9] and from the global RIETE registry [10].

The subgroup analysis of cancer patients in the MATISSE-DVT trial showed that the rates of recurrent VTE in the initial treatment period, for the entire study period and in patients with advanced cancer were as follows for the fondaparinux group versus the enoxaparin group: $2.4 \%$ versus $0.0 \%, 12.7 \%$ versus $5.4 \%$ and $11.5 \%$ versus $3.7 \%$, respectively. On the other hand, major bleeding rates in cancer patients over the entire study period were $7.1 \%$ in the fondaparinux group versus $7.2 \%$ in the enoxaparin group. Thus, this subgroup analysis of cancer patients showed that over the entire study period, there was a trend toward higher rates of recurrent VTE in those patients who were treated with fondaparinux [9].

The Computerised Registry of Patients with Venous Thromboembolism (RIETE registry) has been used to compare the rates of VTE recurrence and bleeding complications in patients with CAT treated with fondaparinux vs. classical antithrombotic treatments at days 10 and 90 . By applying a propensity score-matching analysis, fondaparinux was compared to VKA in patients with and without cancer and to LMWH in those with cancer. Among patients with cancer, there were no differences between fondaparinux and LMWH [10], whereas fondaparinux showed better efficacy than warfarin for preventing VTE recurrence, although this was not statistically significant.

Therefore, globally speaking, the data on fondaparinux are not clear concerning the potential efficacy of this drug for the treatment of VTE in cancer patients.

\section{New data on direct oral anticoagulants (DOACs)}

Several clinical trials have been planned in recent years for this category of drugs, in particular for edoxaban, apixaban and rivaroxaban.

\section{Edoxaban}

Edoxaban was the first direct oral anticoagulant tested in a randomised clinical trial for the treatment of CAT [11]. The HOKUSAICancer trial observed oncology patients with CAT for nearly 12 months. The study was planned to assess whether oral edoxaban is non-inferior to subcutaneous LMWH (i.e. dalteparin) for the treatment of VTE in oncology patients. The primary outcome was the composite endpoint of objectively-confirmed recurrent VTE and major bleeding during the 6-to-12-month study period.

The results reported that oral edoxaban was non-inferior to subcutaneous dalteparin in terms of the composite endpoint of recurrent VTE and major bleeding in CAT. In particular, the rate of recurrent VTE was tendentially lower in patients treated with edoxaban, while the rate of major bleeding was higher with edoxaban than with dalteparin, and this result was mainly influenced by the more frequent occurrence of gastrointestinal bleeding [11].

No significant drug-drug interaction was reported during chemotherapy. Edoxaban dose adjustment was rarely required for patients who developed sudden kidney failure and/or transient thrombocytopaenia with a platelet count below 50,000 per $\mathrm{mm}^{3}$.

\section{Apixaban}

The CARAVAGGIO study is an international clinical trial that was designed to test whether oral apixaban (at a dose of $10 \mathrm{mg}$ twice daily for the first 7 days and then $5 \mathrm{mg}$ twice daily) is non-inferior to subcutaneous dalteparin for the treatment of VTE in patients with cancer [12]. The study is an investigator-initiated, international, prospective, randomised, open-label clinical trial with a non-inferiority endpoint and blind endpoint evaluation (PROBE study). Around 1200 patients are expected to be enrolled in the study and evaluated for a six-month period.

The primary endpoint of the study is objectively-confirmed recurrent VTE as assessed by a central independent adjudication committee that is unaware of the study treatment allocation. The primary safety endpoint is major bleeding, as defined by the guidelines of the International Society of Thrombosis and Haemostasis (ISTH) [12].

This study is ongoing and its results, which could shed more light on the potential effect of DOACs in cancer patients, are expected in early 2020 .

\section{Rivaroxaban}

Rivaroxaban has been tested in this setting in the SELECT D study, which was conducted in the UK. This study enrolled 406 patients with active cancer and VTE who were randomly allocated to receive antithrombotic treatment according to current guidelines (LMWH/ dalteparin) or oral rivaroxaban (15 $\mathrm{mg}$ twice daily for 3 weeks, then $20 \mathrm{mg}$ once daily for a total of 6 months) [13].

The primary endpoint of the study was recurrent VTE, and the secondary safety endpoints were the occurrence of major bleeding and CRNMB. A lower rate (not statistically significant) of recurrent VTE occurred in those patients treated with rivaroxaban. No significant differences in the rate of major bleeding were found between the 
two treatment groups (no episodes of intracranial bleeding), whereas significantly more CRNMB occurred in the rivaroxaban group [13].

The SELECT D study is the first study in the CALLISTO research programme, which aims to evaluate CAT with rivaroxaban. The CALLISTO programme will also include studies that aim to assess the value of this drug for the prophylaxis and treatment of VTE in cancer patients.

\section{Dabigatran}

Currently, no specific randomised trial has compared the efficacy and safety of dabigatran in CAT vs. standard treatment. However, a meta-analysis of the phase III clinical trials RECOVER and RECOVER II provided data concerning the treatment of VTE in CAT with dabigatran or warfarin [14]. As such, only the subgroup of patients with CAT was analysed. The results showed that in cancer patients, dabigatran provided similar clinical benefits to warfarin for VTE recurrence or bleeding. The most significant limitation of this metaanalysis is that it was based on studies that compared dabigatran to warfarin, which is not the gold standard anticoagulant treatment for CAT. Therefore, these data should be confirmed by specific targeted studies to assess the effects of dabigatran in comparison to LMWH.

\section{Conclusion}

In conclusion, there is significant interest in the scientific community regarding the potential use of DOACs in the treatment of $\mathrm{CAT}$, as these drugs would probably be less cumbersome to use than VKAs and more acceptable to patients than long-term subcutaneous LMWH. The first pivotal study data, namely those from the HOKUSAICancer study, seem promising. The results of other, currently ongoing studies such as the CARAVAGGIO study are expected in the near future and could further elucidate the potential value of these drugs for the treatment of CAT, as well as possible differences between the molecules in this pharmacological category.

\section{References}

1. Kuderer NM, Lyman GH (2014) Guidelines for treatment and prevention of venous thromboembolism among patients with cancer. Thromb Res 133: S122-127.

2. Mahé I, Chidiac J, Bertoletti L, Font C, Trujillo-Santos J, et al. (2017) The
Clinical Course of Venous Thromboembolism May Differ According to Cancer Site. Am J Med 130: 337-347.

3. Levine M, Hirsh J, Gent M, Arnold A, Warr D, et al. (1994) Double-blind randomised trial of a very-low-dose warfarin for prevention of thromboembolism in stage IV breast cancer. Lancet 343: 886-889.

4. Moustafa F, Pierfranceschi MG, Di Micco P, Bucherini E, Lorenzo A, et al (2017) Clinical outcomes during anticoagulant therapy in fragile patients with venous thromboembolism. Res Pract Thromb Haemost 1: 172-179.

5. Hutten BA, Prins MH, Gent M, Ginsberg J, Tijssen JG, et al. (2000) Incidence of recurrent thromboembolic and bleeding complications among patients with venous thromboembolism in relation to both malignancy and achieved international normalized ratio: a retrospective analysis. J Clin Oncol 18: 3078 3083.

6. Prandoni P, Lensing AW, Piccioli A, Bernardi E, Simioni P, et al. (2002) Recurrent venous thromboembolism and bleeding complications during anticoagulant treatment in patients with cancer and venous thrombosis. Blood 100: 3484-3488.

7. Lee AY, Levine MN, Baker RI, Bowden C, Kakkar AK, et al. (2003) Lowmolecular-weight heparin versus a coumarin for the prevention of recurrent venous thromboembolism in patients with cancer. N Engl J Med 349: 146-153.

8. Lee AYY, Kamphuisen PW, Meyer G, Bauersachs R, Janas MS, et al. (2015) Tinzaparin vs Warfarin for Treatment of Acute Venous Thromboembolism in Patients with Active Cancer: A Randomized Clinical Trial. JAMA 314: 677-686.

9. Büller HR, Davidson BL, Decousus H, Gallus A, Gent M, et al. (2004) Fondaparinux or enoxaparin for the initial treatment of symptomatic deep venous thrombosis: a randomized trial. Ann Intern Med 140: 867-873.

10. Pesavento R, Amitrano M, Trujillo-Santos J, Di Micco P, Mangiacapra S et al. (2015) Fondaparinux in the initial and long-term treatment of venous thromboembolism. Thromb Res 135: 311-317.

11. Raskob GE, van Es N, Verhamme P, Carrier M, Di Nisio M, et al. (2018) Edoxaban for the treatment of cancer associated venous thromboembolism. N Eng J Med 378: 615-624.

12. Agnelli G, Becattini C, Bauersachs R, Brenner B, Campanini M, et al (2018) Apixaban versus Dalteparin for the Treatment of Acute Venous Thromboembolism in Patients with Cancer: The Caravaggio Study. Thromb Haemost 118: 1668-1678.

13. Young AM, Marshall A, Thirlwall J, Chapman O, Lokare A, et al. (2018) Comparison of an Oral Factor Xa Inhibitor With Low Molecular Weight Heparin in Patients With Cancer With Venous Thromboembolism: Results of a Randomized Trial (SELECT-D). J Clin Oncol 36: 2017-2023.

14. Schulman S, Goldhaber SZ, Kearon C, Kakkar AK, Schellong S, et al. (2015) Treatment with dabigatran or warfarin in patients with venous thromboembolism and cancer. Thromb Haemost 114: 150-157. 